\title{
Design and Implementation of a Real-Time Wireless Sensor Network
}

\author{
J.A. Afonso, H.R. Silva, P.M. Oliveira, J.H. Correia \\ University of Minho \\ Dept. Industrial Electronics \\ 4800-058 Guimaraes, Portugal \\ jose.afonso@dei.uminho.pt
}

\author{
L.A. Rocha \\ Faculty of Eng. University of Porto \\ Dept. Electrical and Computer Eng. \\ 4200-465 Porto, Portugal \\ larocha@fe.up.pt
}

\begin{abstract}
This paper describes the development of wireless sensor network prototype that gathers biometrical data and posture information from several wearable sensor networks and sends it in real-time to a personal computer where the information is monitored and stored. The wireless sensor network is based on a low power real-time MAC protocol that was designed and implemented in the MICAz platform. This paper also presents some analytical results and several experimental results regarding the behavior of the developed system.
\end{abstract}

\section{Introduction}

The evolution of sensor and wireless technologies allows the development of integrated wireless sensor systems in new application areas. An emerging new area of research that combines the strengths and capabilities of electronics and sensors and textiles into one is the field of electronic textiles (e-textiles) [1]. If these e-textiles are further combined with wireless communication capabilities, wireless smart textiles systems with local monitoring and computation skills and remote data storage become reality [2].

This paper describes the development of a system prototype in which biometrical data, posture, goniometric development and position of several individuals, each one wearing a shirt [3] equipped with sensors, can be simultaneously monitored in real-time using a wireless sensor network. An application of this system is the monitoring of teams of athletes in a gymnasium for sports such as basketball, volleyball, handball and gymnastics, with the goal of providing detailed information in order to enhance the performance of both the athletes individually and the team as a whole. Another application is in the medical field, namely in physiotherapy sessions, where such a system can benefit both the patient, by increasing his levels of confidence, and the therapist, by providing detailed information about the patient evolution.

For these applications, a star topology can be used, since all the smart shirts are located near the base station. Each shirt generates periodic traffic several times per second, which requires a MAC protocol capable to provide QoS guarantees (namely low loss and bounded delay) for such traffic. Finally, it is desirable to avoid frequent replacement of the shirt batteries, so their power consumption should be kept to a minimum.

The IEEE 802.15.4 [4] is a wireless personal area network (WPAN) conceived for wireless sensor network applications. The contention based CSMA/CA MAC protocol used by 802.15 .4 is not suitable to provide the QoS required by real-time applications, especially under high loads, due to collisions. The 802.15.4 standard also provides a guaranteed time slot (GTS) scheme in order to support devices requiring dedicated bandwidth or low latency transmission. However, only 7 GTS allocations per superframe are supported with this scheme, and the granularity of transmission time allocations is coarse since each superframe is composed by just 16 slots.

The implementation of our wireless sensor network prototype is based in the CrossBow MICAz [5] platform. MICAz motes are supplied with only the basic functionalities of the 802.15.4 CSMA/CA protocol, but not with the GTS scheme. In order to satisfy our design goals, we conceived and implemented the LPRT MAC protocol. The developed protocol is generic enough to be useful in other realtime wireless sensing and actuation applications.

Next section describes the main components of the developed wireless sensor network. Section 3 presents experimental results gathered from this system while section 4 presents the conclusions. 


\section{The wireless sensor network}

\subsection{Overview}

The developed real-time wireless sensor network is composed by a personal computer (PC), a base station and several wireless smart shirts (stations), as depicted in Fig. 1. Each shirt aggregates data from a heart rate sensor, a respiratory sensor and several posture sensors, sending continuously the information from its multiple sensors to the base station. The posture system enables the measurement of the angles of rotation of spine and arms. Microcontroller, power supply, RF transceiver and antenna are also attached to the shirt. The base station is connected to a PC, where the data collected from the shirts can be stored and visualized by the operator in real-time. A positioning system was not implemented yet.

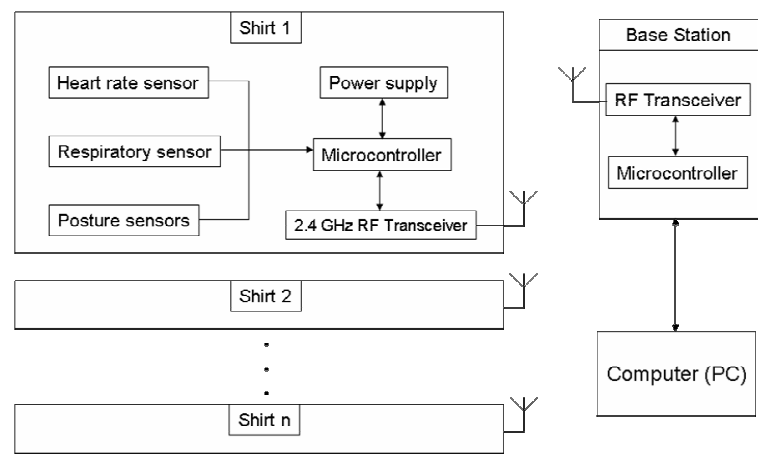

Figure 1. Schematic of the wireless sensor network.

Both base station and shirt stations are implemented using MICAz motes. The base station is attached to a MIB520 interfacing and programming board, which communicates with and receives power from the PC through a USB cable. The shirt stations are powered by two AA batteries.

\subsection{The LPRT protocol}

The LPRT protocol was implemented in the MICAz motes using the TinyOS operating system and the nesC programming language [6]. It is a hybrid schedule based dynamic TDMA protocol and contention based CSMA/CA protocol. The LPRT protocol defines a superframe structure as presented in Fig. 2. Each superframe is divided in a fixed number of mini-slots (1024, in the current implementation), and starts with the transmission, by the base station, of the respective beacon frame (B), which is followed by the Contention Period $(\mathrm{CP})$. During the $\mathrm{CP}$ any station can transmit using the rules of a CSMA/CA protocol. The $\mathrm{CP}$ allows the stations to associate with the base station and to request mini-slots for transmission during the Contention Free Period (CFP). It can also used to convey non-real-time asynchronous traffic. Stations must not initiate a CSMA/CA transaction if it cannot be completed before the beginning of the CFP.

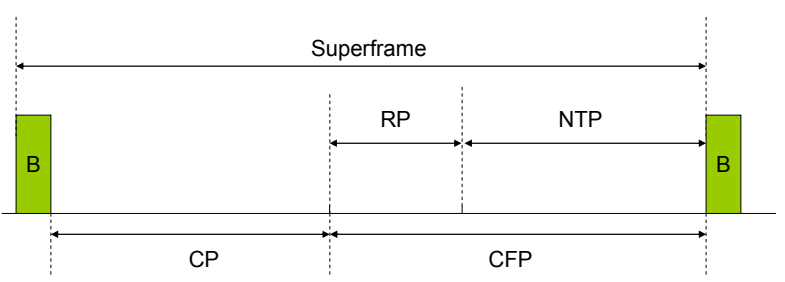

Figure 2. Superframe format of the LPRT protocol.

The Contention Free Period is placed after the CP. Transmissions during the CFP are scheduled by the base station using resource grant (RG) information announced previously in the beacon frame of the current superframe. Since transmissions during the CFP are regulated by the base station, they are not affected by the hidden station problem, unlike transmissions of protocols that rely on carrier sensing.

The CFP is composed by an optional retransmission period (RP) and a normal transmission period (NTP). The RP gives a new opportunity for transmission of data frame when it was not delivered correctly in the previous superframe. The rationale of placing the RP before the NTP is to allow the data frame to reach its destination before the next one is supposed to be transmitted, in order to limit the delay and preserve the sequence of data. This division is not mandatory, however, as retransmissions can be mixed with regular transmissions during the entire CFP period. The retransmission procedure is intended to increase the reliability of the protocol, which is fundamental for applications with low loss tolerance.

$\mathrm{MICAz}$ motes use fixed 16 bit long station addresses. Instead of using these addresses in the resource grant (RG) fields of the beacon to identify the stations that are allowed to transmit during the CFP, the LPRT protocol uses a smaller association ID (AID) that is dynamically assigned to the stations when they associate with the base station. This approach reduces the length required for the beacon, making it less sensible to channel errors. The current implementation uses 5 bit long AID addresses, allowing a maximum of 32 associated stations, which is sufficient for the envisioned applications.

Figure 3 shows the structure of the payload of the beacon frame. The superframe duration field gives the duration of the current superframe in multiples of a minimum superframe duration time. It is followed by a 
list of resource grant ( $R G$ ) fields, whose quantity is expressed by the RG list length (RLL) field. Each RG is composed by a transmission direction (TD) bit, the association ID (AID) field and an initial transmission slot (ITS) field. The RG allows the scheduling of data transmissions either to or from the station identified by the AID, depending of the value of the TD bit: " 0 " for downlink and " 1 " for uplink direction. More than one RG can be granted to a station in the same superframe. The total transmission period granted by a given $R G$ goes from the beginning of respective ITS until before the beginning of the ITS of the next RG on the list. The uplink data can include piggybacked information for alteration of resource allocation parameters, if desired.

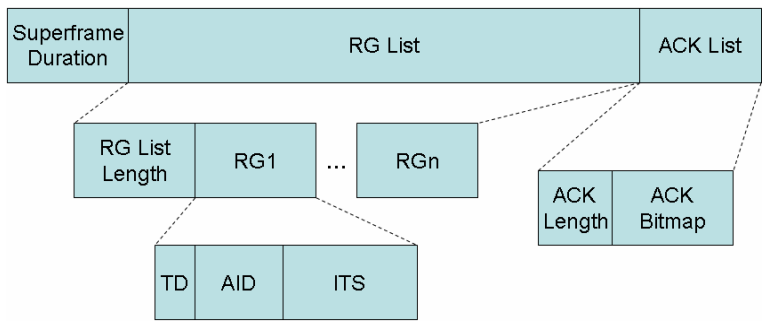

Figure 3. Structure of the beacon frame payload.

For downlink transmissions the ACK frame follows the data transmission, while for uplink transmissions the acknowledgment is made using the ACK list of the next beacon frame. The piggybacking of acknowledgments in the beacon frame eliminates the power consumption and overhead associated with the reception of individual ACK frames for each uplink data frame. The ACK list is composed by an ACK length (AL) field and an ACK bitmap field containing one bit for each uplink RG of the previous superframe. A successful transmission is indicated by a " 1 " in the respective bitmap position, while a lost or corrupted transmission is indicated by a " 0 ".

To reduce the power consumption of the stations, after a station listen to the beacon, it turns off the radio transceiver until the moment of a scheduled transmission or reception of data, after which it turns off the transceiver again. The station switches on the transceiver again at the beginning of the next superframe to receive a new beacon, and the cycle repeats. Each time the station receives a beacon it resynchronizes its clock with the base station.

\subsection{Protocol analysis}

This section presents a simple analysis of the LPRT protocol with uplink data traffic, assuming an
AWGN/BSC channel, which is later used for comparison with experimental data.

The packet error rate $\left(P E R_{D}\right)$ for an uplink data transmission is given by:

$$
P E R_{D}=1-(1-B E R)^{L_{D}},
$$

where $L_{D}$ is the length of the data packet in bits and $B E R$ is the channel bit error rate. The packet error rate $\left(P E R_{B}\right)$ for a beacon transmission, with $L_{B}$ as the beacon length in bits, is calculated in a similar way.

The delivery error rate (DER) is defined here as the probability of failure in the delivery of a data message from a given station to the base station. We consider that one data message (e.g., a sample from all sensors of a smart shirt) in generated at each superframe. For the LPRT protocol, the basic frame exchange transaction required for the delivery of an uplink data message consists of the transmission by the base station of a beacon frame at the start of a superframe followed by the transmission by a station of a data frame, at the position announced by the beacon, in the same superframe.

A successful transaction in this case requires the successful transmission of both the beacon and the data frame; therefore the delivery error rate in this case $\left(D E R_{0}\right)$ is:

$$
D E R_{0}=1-\left(1-P E R_{B}\right)\left(1-P E R_{D}\right) .
$$

In order to increase the robustness of the communications, the LPRT protocol implements a simple retransmission mechanism that works by automatically rescheduling the transmission of a data message in the next superframe whenever the transaction in the current superframe fails. If the attempt of delivery of the data message fails in two consecutive superframes the message is dropped. The improved delivery error rate with retransmissions is:

$$
D E R_{1}=D E R_{0}{ }^{2} \text {. }
$$

One can notice that the rescheduled transmission can be either a retransmission, if the data transmission in the previous superframe failed, or the first transmission, if instead the beacon in the previously superframe was not received, since it precludes the respective data transmission. For the sake of simplicity, however, the rescheduled transmission will continue to be referred here as a retransmission.

The sensors samples included in the data message are taken just before its transmission, so the delay without retransmission is almost zero, while the maximum increment in delay for a retransmitted data message is less than one superframe period. 
The average current consumption of the LPRT protocol without retransmissions $\left(I_{0}\right)$ is given by:

$$
I_{0}=\frac{T_{B}+G_{B}+T_{D}+G_{D}}{T_{S F}}\left(I_{O N}-I_{O F F}\right)+I_{O F F}
$$

where $T_{B}$ and $T_{D}$ are the beacon and data transmission times, $G_{B}$ and $G_{D}$ are the respective guard times, $I_{O N}$ is the current consumption in the active state and $I_{O F F}$ is the consumption in the sleep state. The guard times are the periods between the transition to the active state and either the effective transmission of data or the reception of the beacon.

The average current consumption with retransmissions increases by a factor that depends on the delivery error rate, and is given by:

$$
I_{1}=I_{0}+\frac{T_{D}+G_{D}}{T_{S F}}\left(I_{O N}-I_{O F F}\right) B E R_{0}
$$

\subsection{PC application}

The TinyOS installation CD includes a java application called SerialForwarder that reads packets from the serial port and forwards those through a given TPC port to a network client application, and vice versa. Based on this java application, we developed an application that introduces several new functionalities. It provides information about the state of the connection between the base station and each station in real-time (Fig. 4), including the number of beacons not received by a station and the number of data frames not received by the base station, as well as the delivery error rate without retransmissions and with retransmissions. The user interface presents this data both for the current values (evaluated from the last $N$ samples) and cumulative values (since the start of data collection). The receiver signal strength indicator (RSSI) and the battery voltage of the station are also presented. The data can also be registered in a file for posterior analysis.

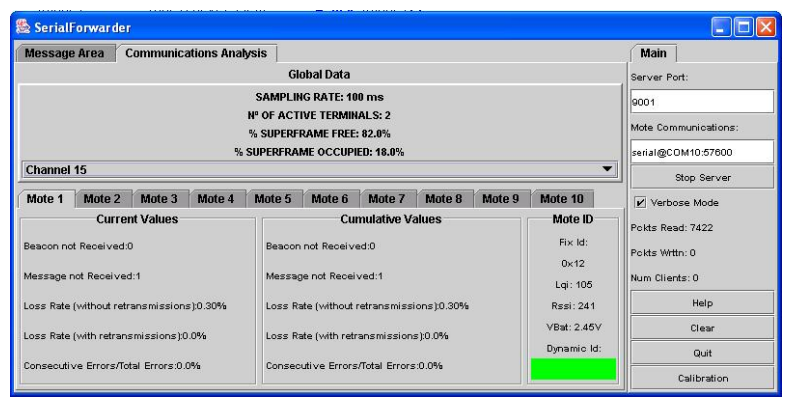

Figure 4. Main user interface.
The developed application receives the raw posture sensors data, performs the calculation of the angles of the articulations and forwards this information to the client application, which acts as a data base and allows 3D visualization of data. It also includes a functionality that allows the sensors to be remotely calibrated though the wireless sensor network (Fig. 5), thereby avoiding the requirement of physical connection of the data station to a programming board.

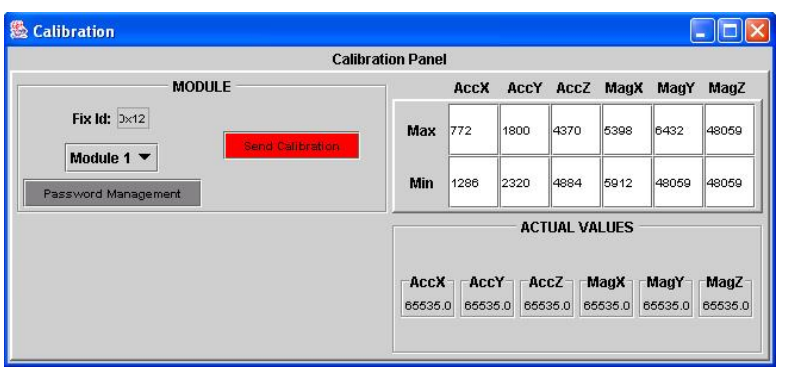

Figure 5. Calibration interface.

\section{Experimental results}

First results present the current consumption of the MICAz motes as a function of time, which include both the consumption of the transceiver and of the microcontroller. The evaluation scenario is composed by a base station and four stations. Each station collects 36 byte samples periodically, with a sampling rate of $5 \mathrm{~Hz}$, which corresponds to a packet interarrival time of $200 \mathrm{~ms}$. The superframe period was chosen to be equal to the packet inter-arrival time, so one sample is transmitted per superframe. Figure 6 presents the current consumption for one of the stations. The current consumption of the microcontroller $\left(I_{O F F}\right)$ is $8 \mathrm{~mA}$ while the consumption with the transceiver turned on $\left(I_{O N}\right)$ is around $28 \mathrm{~mA}$.

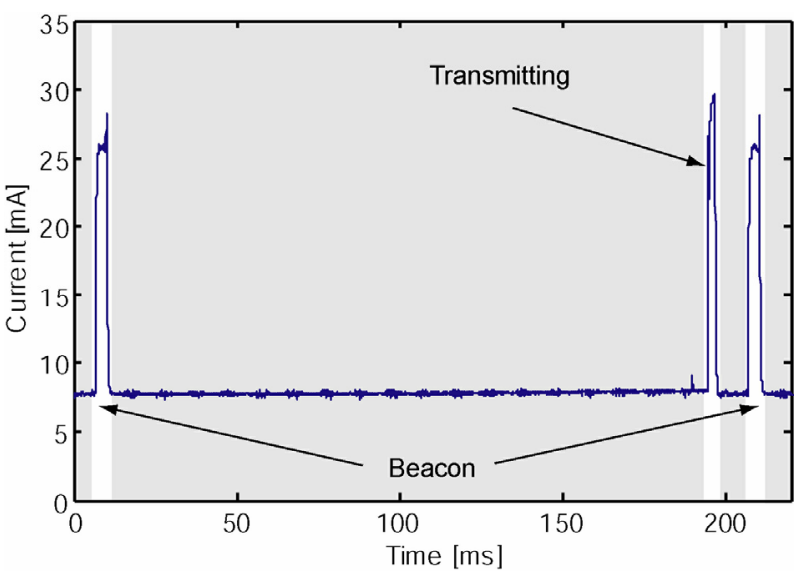

Figure 6. Current consumption of the LPRT protocol as a function of time for one particular station. 
Figure 7 presents a closer look to the current consumption charts of the four stations in the RG list, superimposed, where the transmission of each station and the reception of the beacon of the next superframe can be seen. Each superframe is divided in 1024 minislots, allowing a high level of granularity in the allocation of transmission time to the stations. With these data frame size and without channel errors, the LPRT protocol allows up to 60 stations per superframe. It can achieve high throughput with high loads without increase in delay or losses by collision, unlike contention based protocols.

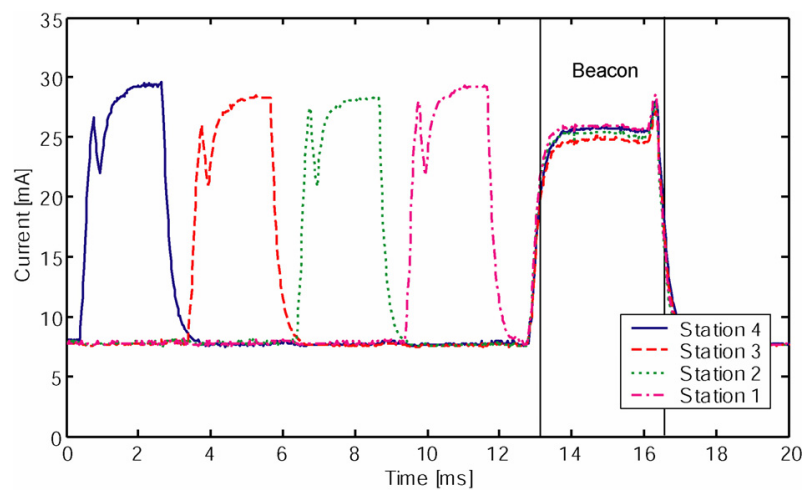

Figure 7. Current consumption of the LPRT protocol as a function of time for four stations.

The evaluation setup for the second experiment is composed by two networks operating in different channels (11 and 26) at the same time in the same place. Each network is composed by only one base station and one station located 3 meters apart and using the same transmission power $(0 \mathrm{dBm})$. The superframe period is $T_{S F}=100 \mathrm{~ms}$. The samples are 75 bytes long, so the data frame length, including headers is $L_{D}=712$ bits while the beacon length is $L_{B}=160$ bits, which means that the respective transmission times, given that the network data rate is $250 \mathrm{kbit} / \mathrm{s}$, are $T_{D}=2.85$ $\mathrm{ms}$ and $T_{B}=0.64 \mathrm{~ms}$.

Each station was powered by two $2300 \mathrm{mAh} \mathrm{Ni}$ MH AA batteries. Both networks started to operate in a Thursday at 17:00h. The channel 11 network ceased to operate when the batteries drained out. The operation of channel 26 network lasted longer but was terminated due to a power outage in the laboratory.

MICAz uses the same channels of $2.4 \mathrm{GHz}$ version of 802.15.4. Center frequency for channel 11 is 2405 $\mathrm{MHz}$, while for channel 26 the center frequency is $2480 \mathrm{MHz}$. Channel width is $5 \mathrm{MHz}$.

The signals of three 802.11 access points operating on channel 1 were detected at the place of the experiment. Channel 1 is centered on $2412 \mathrm{MHz}$ and has a width of $22 \mathrm{MHz}$, so it overlaps with channel 11 of 802.15.4. 802.11 access points operating in other channels were also detected, but there were no overlapping with 802.15.4 channel 26 .

Figure 8 presents the delivery error rate as a function of time calculated using the previous 1000 samples. Asterisks in the graph identify disconnections, triggered by several consecutive missing data frames, followed by new associations. The number of errors for the network operating in channel 11 was significantly higher (overall delivery error rate without retransmissions was $0.66 \%$ for channel 11 and $0.02 \%$ for channel 26). These results can be explained by interference from nearby 802.11 networks. In fact, the great majority of errors occurred during working hours.

In order to avoid interference, the user interface of the developed PC application allows the operator to change the operating channel (Fig. 4). The information about the new channel is sent on the beacon, allowing a seamless channel transition for the stations. Since the state of the links is monitored in real-time by the application, an automatic channel selection mechanism could be easily implemented as well.

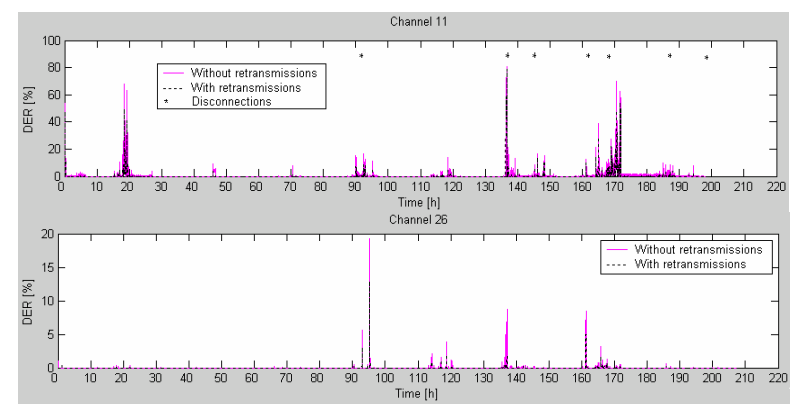

Figure 8. Delivery error rate as a function of time.

Figure 9 presents the relation between the delivery error rate with and without retransmissions, comparing the experimental data for channel 11 with the analytical data (equation 3). Even though there is a set of data points around the analytical curve, there is another set of points for which the efficacy of the retransmission mechanism is almost none, so the overall performance is worst then expected. Further analysis needs to take in account another effects such as burst errors due to fading.

Figure 10 presents the battery voltage as function of time. It drops quickly when the voltage reaches around $2.2 \mathrm{~V}$. The guard times that were used are $G_{D}=1 \mathrm{~ms}$ and $G_{B}=3.2 \mathrm{~ms}$, thus, according to equation 4 , the average current consumption without retransmissions is $I_{0}=9.54 \mathrm{~mA}$, so the theoretical autonomy of the station with fully charged $2300 \mathrm{mAh}$ batteries is $241 \mathrm{~h}$. An inspection of the channel 11 chart indicates that the 
autonomy of the channel 26 station would probably be around $230 \mathrm{~h}$, which is close to the theoretical value, if it was not for the power outage. The autonomy of the channel 11 station was lower, probably because its batteries were not with full capacity anymore, since the increment in consumption due to retransmissions (equation 5) is small. Even in the worst case $\left(D E R_{0}=\right.$ 1) it would be only $8 \%$.

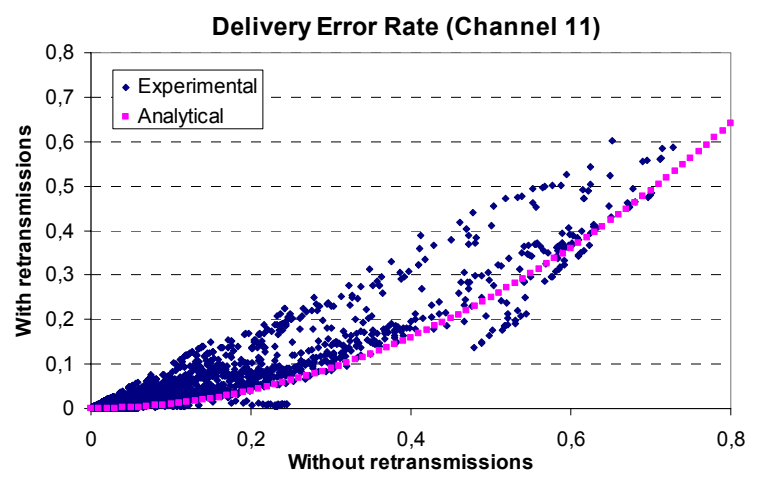

Figure 9. DER with and without retransmissions.

The average current consumption of stations could be reduced to $2.15 \mathrm{~mA}$ if the microcontroller was turned off during the sleep state, increasing significantly the autonomy.

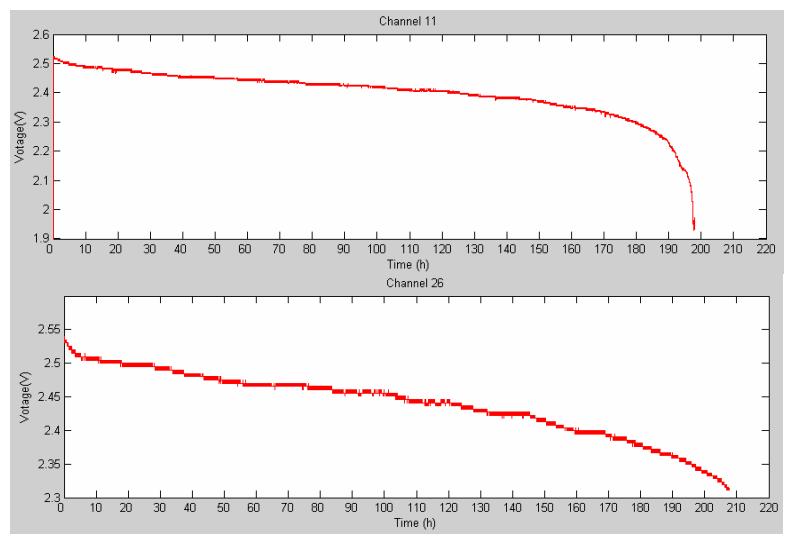

Figure 10. Battery voltage as function of time.

Another set of experiments was made in a hall with one base station and one station. A range $(r)$ of $30 \mathrm{~m}$ with moderate channel errors $\left(B E R_{0}<10 \%\right)$ was achieved when both the base station and the station were at the chest level. However, when the station was placed at ground level, the errors increased significantly and disconnections were frequent, even for shorter distances. This behavior can be explained by the radio signal power dropping off with $r^{4}$ [7] due to ground reflections for short antenna heights.
When the base station was placed over the station, the channel conditions were not affected by the height of the station antenna, even when the station was placed at the ground. This results lead to conclusion that a good place to put the base station is at the ceiling, in the middle of the hall, because that way it is located at a center position related to the stations and it is less affected by the height of the stations antennas.

\section{Conclusions}

This paper described a real-time wireless sensor network implemented using the MICAz platform that collects data from several smart shirts simultaneously. The developed system includes a low power real time MAC protocol, a retransmission mechanism to increase the robustness of communications, a PC application that monitors the link errors in real-time, a user interface for remote calibration of the sensors on the shirts and a channel selection mechanism to cope with interference.

According to the experimental results, the range of the network, the autonomy of the shirts and the number of supported stations are compatible with the requirements of the target applications.

\section{References}

[1] D. Marculesco et al., "Ready to ware", IEEE Spectrum, pp. 29-32, October 2003.

[2] M. Catrysse et al., "Towards the integration of textile sensors in a wireless monitoring suit", Sensors and Actuations A, 114, pp. 302-311, 2004.

[3] J. P. Carmo, P. M. Mendes, C. Couto and J. H. Correia, "2.4 GHz wireless sensor network for smart electronic shirts", SPIE Microtechnologies for the New Millennium 2005, pp. 579-586, Sevilla, Spain, May 2005.

[4] IEEE Std 802.15.4-2003, Wireless Medium Access Control (MAC) and Physical Layer (PHY) Specifications for Low-Rate Wireless Personal Area Networks (LR-WPANs), October 2003.

[5] Crossbow Technology, Inc: MPR/MIB User's Manual, April 2005, available at http://www.xbow.com .

[6] D. Gay et al., "The nesC language: A holistic approach to networked embedded systems", ACM SIGPLAN 2003 conference on Programming language design and implementation, pp. 1-11, San Diego, California, USA, June 2003.

[7] D. Estrin, L. Girod, G. Pottie and M. Srivastava, "Instrumenting the world with wireless sensor networks", IEEE International Conference on Acoustics, Speech, and Signal Processing (ICASSP '01), Salt Lake City, USA, May 2001 